\title{
Influence of Uncoupled Diblock Molecules on Mechanical Properties of Styerene/Butadiene/ Styrene Triblock Copolymers
}

\author{
Rameshwar Adhikari \\ Central Department of Chemistry, Tribhuvan University, Kirtipur, Kathmandu \\ e-mail: nepalpolymer@yahoo.com
}

\begin{abstract}
The influence of the presence of uncoupled polystyrene-block-polybutadiene (SB) diblock chains to polystyreneblock-polybutadiene-block-polystyrene (SBS) triblock copolymers on the mechanical properties of the latter has been studied by means of tensile testing and dynamic mechanical analysis preparing several lamellae forming SBS/ SB blends through solution casting. The microphase-separated morphology of the samples was investigated by transmission electron microscopy. Both large strain deformation tensile deformation behaviour and viscoelastic properties of the SBS block copolymers were found to be affected appreciably by the presence of uncoupled SB diblock. The storage modulus of linear SBS was found to drop more sharply in the plateau region than for the radial SBS at the same SB content. At low SB content (up to $20 \mathrm{wt} .-\%$ for linear SBS and still higher for radial one), the overall tensile properties was not negatively influenced. On the whole, star block copolymers were found to be less sensitive towards the presence of diblock.
\end{abstract}

Key words: block copolymer, nanostructure, mechanical properties, electron microscopy

\section{Introduction}

Block copolymers (BCPs) constitute a special class of heterogeneous polymers in which two or more homopolymer chains (blocks) exist in a single molecule (Figure 1). The incompatibility of the constituent blocks leads to intramolecular phase-separation but the chemical connectivity restricts the spatial dimension of phase segregation to nanoscale. As a result, at sufficiently high molecular weight, monodisperse BCPs form an array of periodic nanostructures (periodicity $10-100 \mathrm{~nm}$ ) that are commonly referred to as microphase-separated structures (Hamley 1998). In fact, the individual nanodomains thus formed preserve the properties of the respective homopolymers. By changing the relative composition and the degree of compatibility of the component polymers, the size and the type of nanostructures can be precisely controlled (Hamley 1998, Bates \& Fredrickson 1990). More recent works have shown that the morphologies and hence the mechanical behaviour of block copolymers systems are further influenced by other molecular parameters such as chain architecture, bridge/loop conformation ratio, etc. (Adhikari \& Michler 2004, Matsen 2000, Milner 1994, Drolet and Fredrickson 1999). 


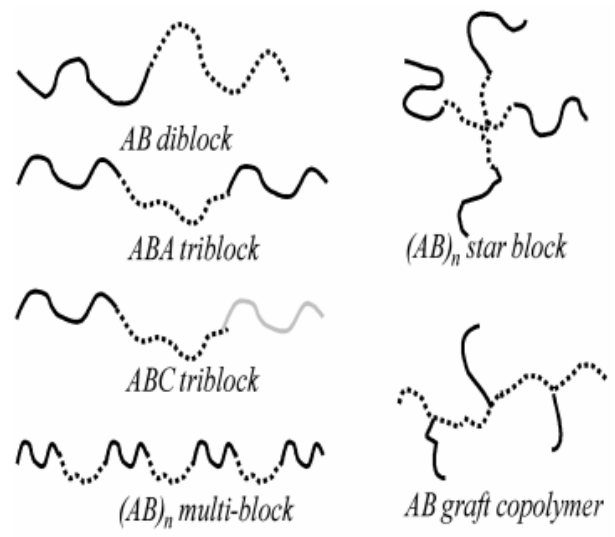

Fig 1. Architectures of frequently studied blockcopolymers

Generally speaking there are two kinds of BCPs with respect to their molecular construction (see Figure 1, Adhikari 2012): linear and branched. The linear ones can be $\mathrm{AB}$ diblocks, $\mathrm{ABA}$ triblocks, $\mathrm{ABC}$ triblocks, $(A B)_{n}$ multiblocks etc. (where the letters A and B stand for individual polymer blocks). Graft copolymers and star shaped (also called radial) ones belong to the branched category. The tailored BCPs are generally synthesized by ionic polymerisation (Quirk \& Morton 1998). However, recently controlled radical polymerisation has been successfully used to synthesize well-defined BCP architectures (Davis \& Matyjaszewski 2002).

Polystyrene-block-polybutadiene-block-polystyrene (SBS) triblock copolymers are thermoplastic elastomers, i.e., these act as rubbers or elastomers at room temperature while, unlike other elastomers, can be processed in the same manner as thermoplastics at elevated temperatures. The reason behind is the phase segregated nanostructures of the copolymer in which the highly flexible polybutadiene (PB) chains (glass transition temperature, $\mathrm{T}_{\mathrm{g}}$ ca. $-100{ }^{\circ} \mathrm{C}$ ) are crosslinked by glass-like stiff polystyrene (PS) chains (glass transition temperature, $\mathrm{T}_{\mathrm{g}} \mathrm{ca} .+100^{\circ} \mathrm{C}$ ). Generally, the SBS thermoplastic elastomers comprise 20-30 wt.-\% PS and rest PB. In the solid state, PS and PB chains undergo intra-molecular phase separation and form periodic cylindrical nanostructures when PS/PB composition is $30 / 70$ (see Fig. 2).

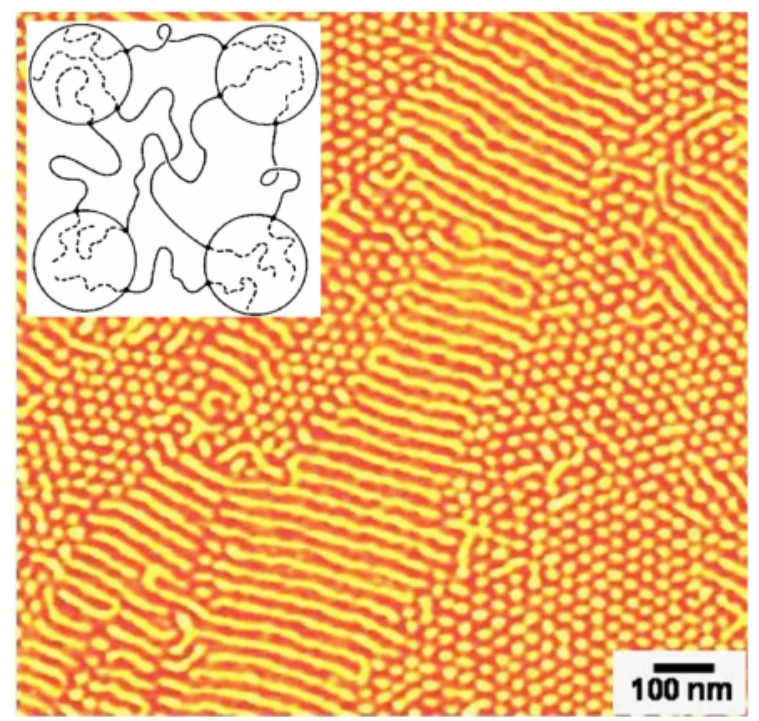

Fig. 2. Atomic force micrographs of an SBS triblock copolymer comprising $30 \mathrm{wt} .-\%$ PS; the inset shows schematic representation of intra-molecular phase segregation leading to the formation of periodically arranged PS nanodomains; note that the scale bar is $100 \mathrm{~nm}$.

Upon cooling from the molten state, the glassy PS domains of the copolymer get vitrified at the vicinity of $\mathrm{T}_{\mathrm{g}}$ and act as physical cross-links. On increasing the temperatures, these domains again dissolve destroying the cross-links and enabling remoulding of the polymers. It should be kept in mind that the nature of the nanostructures formed (which depends on the composition of the triblock copolymer) determines mechanical properties of the block copolymers (Holden 2000).

One of the common methods of synthesis of the SBS triblock copolymers comprises the sequential polymerisation of polystyrene (PS) block attached to polybutadiene (PB) chain and subsequent coupling of resulting molecules at reactive $\mathrm{PB}$ chain ends (Quirk \& Morton 1998). This gives rise finally to an SBS triblock copolymer. However, depending on the efficiency of the coupling agent as well as other experimental conditions, a fraction of SB diblock molecules may remain uncoupled leading to SBS/SB mixtures. The presence of SB molecules changes neither the effective volume fraction and nor the curvature of interface. The respective chains of the diblock molecules are mixed to the corresponding 
domains of the triblock copolymers. As a result, the overall morphology of the block copolymer also remains unaltered. Thus, at low SB content, one may expect that the mechanical properties are not significantly affected.

There have been some recent works on the viscoelastic properties of low styrene content SBS block copolymers containing SB diblock molecules. It has been claimed that the pure triblock copolymers have far better properties than those containing SB diblock molecules (McKay et al. 1995, Lovisi et al. 1998). Guided by these notions, even some styrenic triblock copolymers containing no diblock have even been commercialised (McKay et al. 1995).

Recently, we studied the effect of molecular coupling on the mechanical properties of styrenic block copolymers using blends of the block copolymers having different morphologies and net chemical compositions (Adhikari et al. 2010). It was demonstrated that the mechanical properties of the blends are simple functions of blends composition as long as the molecular coupling of the outer glassy block are guaranteed in such block copolymer. In the present work, we keep the net chemical composition and morphology of the blends constant and vary the amount of molecular coupling by introducing diblock chains into the domains of the triblock copolymer. The aim of the work is to analyse the impact of SB diblock molecules on the overall mechanical properties of SBS block copolymer systems especially at high strains. The effect of diblock copolymer is also investigated taking into account the molecular topology of the triblock copolymer. For preparing the blends, the PS/ PB composition of the triblock and diblock copolymers chosen is around 50/50 (lamellar morphology). This composition is commonly attractive for the applications like compounding with general purpose polystyrene (GPPS) and as thermoplastic elastomers.

\section{Methodology \\ Experimental Section \\ Materials and sample preparation}

The block copolymers used in this study are listed in Table 1. Different SBS/SB mixtures were prepared by solution blending using toluene as solvent. Films approximately $1 \mathrm{~mm}$ thick were prepared by allowing the solvent to evaporate over a period of about 10 days. The resulting films were dried for several days and finally annealed at $120^{\circ} \mathrm{C}$ for 48 hours in vacuum. The blends contained 10, 15, 20, 30, 40 and 60 wt.- $\%$ SB diblock.

Table 1. Characteristics of the block copolymers used in this study

\begin{tabular}{ccccl}
\hline Polymer & Architecture & PS/PB composition & Diblock \% & Morphology expected \\
\hline SBS-L1 & linear & $50 / 50$ & 0 & lamellae \\
SBS-S2 & radial & $50 / 50$ & - & lamellae \\
SBS-L3 & linear & $30 / 70$ & 0 & PS cylinder \\
SBS-S4 & radial & $30 / 70$ & - & PS cylinders \\
SB & linear & $50 / 50$ & 100 & lamellae \\
\hline
\end{tabular}

$L$ - linear triblock, $S$ - star block copolymer; the PS/PB weight fractions are mentioned as 50/50 and 30/70

\section{Characterisation methods}

The methods used are tensile testing, dynamic mechanical analysis (DMA) and transmission electron microscopy (TEM). For the mechanical characterisation using universal tensile machine, $50 \mathrm{~mm}$ long dog-bone shaped tensile bars were punched out of the films of each sample. The bars were strained at a cross head speed of $50 \mathrm{~mm} / \mathrm{min}$ at $23^{\circ} \mathrm{C}$. At least 5 specimens each of the sample were strained up to break. DMA measurements were performed on solution cast films over the temperature range of $-150^{\circ} \mathrm{C}$ to $+150^{\circ} \mathrm{C}$ at 1 $\mathrm{Hz}$. For the TEM analysis, thin sections of the samples, approximately $70 \mathrm{~nm}$ thick, were prepared by cryoultramicrotomy followed by treatment of the sections by osmium tetroxide $\left(\mathrm{OsO}_{4}\right)$ vapour so that double bond containing butadiene phase of the block copolymer gets selectively stained and appear dark in the TEM micrographs.

\section{Results and Discussion \\ Morphological characterisation}

For the samples SBS-L1 and SBS-S2, lamellar morphology was observed as expected from their composition (PS/PB ratio of 50/50). For example, transmission electron micrographs of the sample SBS2 is shown in Figure 3. Sample SB-L1 should also possess a lamellar morphology similar to SBS-L1 and SBS-L2 owing to its PS/PB ratio of 50/50. 


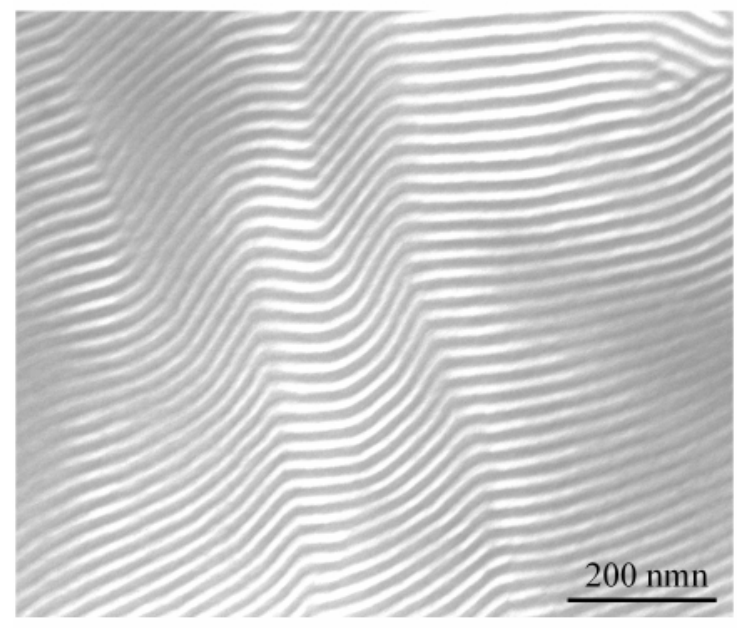

Fig. 3. TEM micrograph of the sample SBS-S2 (PS/PB composition 50/50); $\mathrm{PB}$ phase is stained by $\mathrm{OsO}_{4}$ (appearing dark); similar morphology observed for SBS-L1.

Sample SBS-L3, a linear block copolymer with $30 \mathrm{wt}$.$\%$ PS exhibits cylindrical morphology with PS cylinders dispersed in PB matrix (similar to one shown in Figure 2). With equivalent PS content, similar morphology can be expected for SBS-S4. However, co-continuous morphology was observed which closely resembled Gyroid morphology of the block copolymer discussed in the literature (see Figure 4).

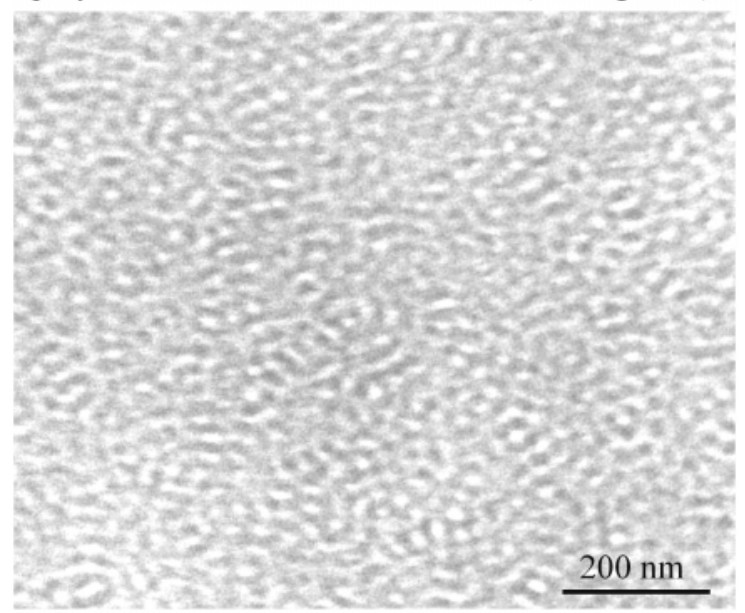

Fig. 4. TEM micrograph of low styrene content SBS block copolymers (PS/PB composition approximately 30/70; i.e. SBS-S4) showing 'Gyroid' morphology; PB phase is stained by $\mathrm{OsO}_{4}$ (appearing dark).
The formation stable co-continuous Gyroid morphology in the radial SBS block copolymer was further confirmed by repeating the sample preparation and investigation by the transmission electron microscopy (TEM). However, the PS composition range is quite different from the literature value ( $33 \%$ for Gyroid morphology; Hamley 1998). To verify whether the interfacial curvature change leading to the shift in the phase behaviour is due to radial geometry, PS/PB composition should be independently checked again. Actually, the presence of Gyroid morphology is further supported by its peculiar tensile behaviour (see next section).

\section{Stress-strain behaviour of the block copolymers}

Tensile stress-strain curves of the triblock copolymers studied (both linear and radial ones) are presented in Figure 5. All the samples show highly ductile behaviour as demostrated by a large deformation (several hundred percent elongations) up to break.

As usual for the lamellar block copolymer, the deformation behaviour of samples SBS-L1 and SBSS2 is characterised by a well defined yield point and a large plastic deformation followed by strain hardening at large strain. SBS-L3 behaves like a thermoplastic elastomer as known in the literature. The radial block copolymer (SBS-S4), with the net chemical composition identical to that of SBS-L3, shows an interesting phenomenon, i.e. its deformation is accompanied by yielding process (pronounced necking and drawing of tensile specimen). Its properties are far better (much higher tensile strength) than that of SBS-L3. The yielding and drawing behaviour of our star shaped copolymer under tensile deformation is in line with the earlier works on other linear styrenic triblock copolymer having Gyroid morphology (Dair et al. 1999, Honeker and Thomas 2000). Thus the typical mechanical performance of the SBS-S4 also supports the presence of Gyroid morphology.

Furthermore, independent of the total PS/PB composition, the block copolymers having star architectures (SBS-S2 and SBS-S4) show higher tensile strengths than their linear analogues (SBS-L1 and SBSL3) whereas their strain at break is comparable. Figure 5 demonstrates nicely the fact that the star copolymers exhibit better mechanical properties than their linear analogues. The effect can be explained by the presence 
of larger number of physical cross-linking sites in the radial copolymer chains than in the linear triblocks (Holden 2000, Quirk and Morton 1998).

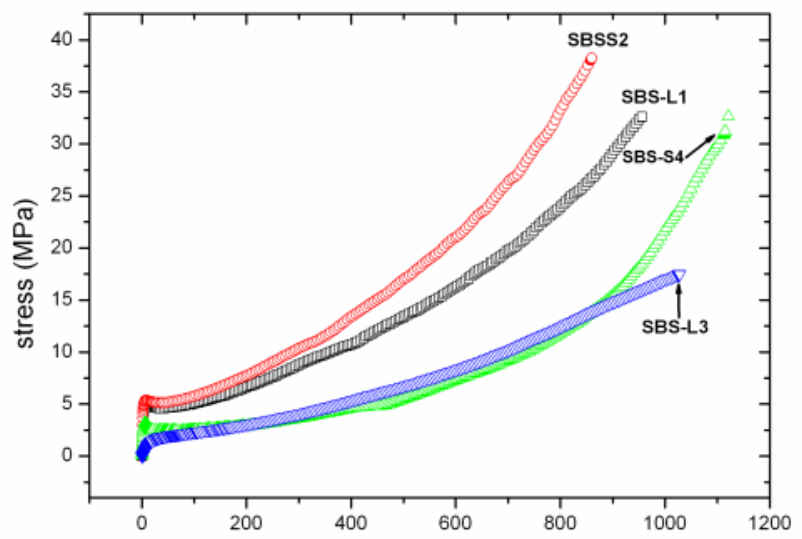

Fig. 5. Representative stress-strain curves of the SBS block copolymers studied, tensile testing at $23^{\circ} \mathrm{C}$ on solution cast films.

\section{Deformation behaviour of SBS/SB mixtures}

Let us examine now the tensile properties of SBS/SB mixtures (i.e. the blends of SB diblock with SBS-L1 and SBS-S2) where the overall PS/PB composition remains practically constant. All the blends have identical PS/PB composition (nearly 50/50) and thus should possess lamellar morphology. The only thing that changes is the amount of uncoupled molecules which increases with addition of SB diblock to the SBS triblock or star shaped copolymers. The SB diblock has butadiene block on one side and styrene block on the other. Hence, the glassy PS chains of the diblock are coupled with other PS block of the SBS triblock copolymer while its highly flexible PB blocks remain dangled and uncoupled.

For the blends with SBS-L1 (see Figure 6a), up to 20 wt.- $\%$ of SB diblock, the yield stress of the samples is not affected by the addition of SB molecules. At higher deformation (> about $200 \%$ strain), the stress level at every strain drops slightly. There is small decrease in tensile strength (stress at break) but the strain at break significantly increases. If we consider the area under the stress-strain curves as a measure of toughness, the blends are as tough as the pure SBS up to about 30 wt.-\% SB. At higher SB concentration (>30 wt.-\%), the tensile stress drops rapidly.

Stress-strain curves of the blends of SB diblock copolymer with radial triblock copolymer (SBS-S2) are presented in Figure 6b. Actually, the neat star block copolymer already contains about 14 wt.- $\%$ uncoupled SB diblock but still shows the tensile stress much higher than that of linear SBS block copolymer (SBS-L1), see also Figure 5. As in the case of linear SBS-L1/SB mixtures, the decrease in tensile strength occurs by further addition of SB diblock. At higher SB content (additional 20-40 wt.-\% SB), the blends have reasonably high level of tensile strength and large elongation at break. In the blends with star block copolymer the drastic drop in stress level appears at much higher SB content than in the corresponding blends with linear block copolymer. Obviously, the properties of radial copolymer are less sensitive towards the uncoupled diblock molecules.

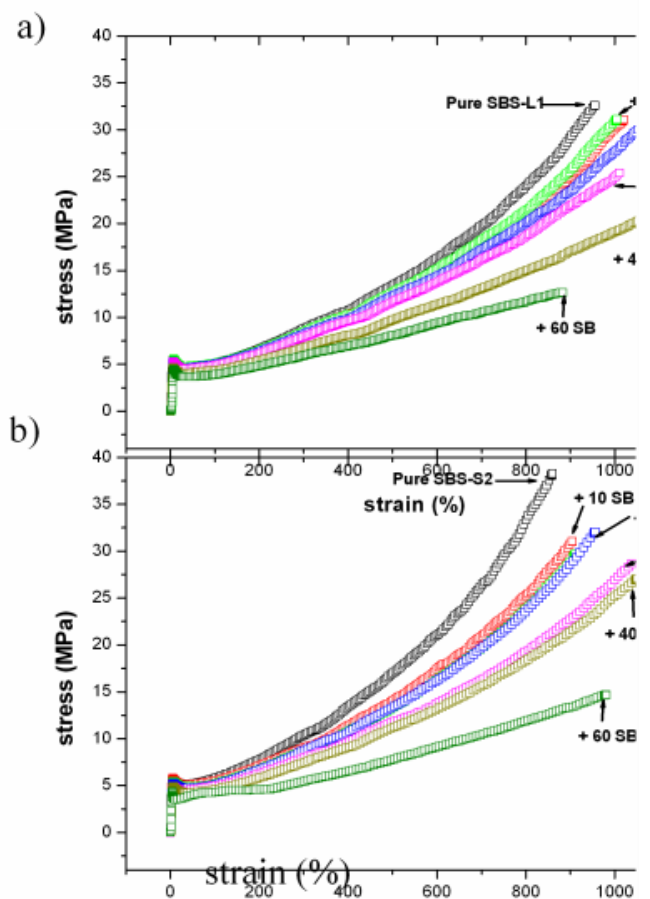

Fig. 6. Stress-strain curves of lamellar block copolymers containing various amount of SB diblock molecules, tensile testing at $23^{\circ} \mathrm{C}$ on solution cast films: a) SBS-L1/SB blends and b) SBS-S2/SB blends.

On adding the SB diblock copolymer (PS/PB composition approximately 50/50) to the low styrene SBS triblock copolymers (namely SBS-L3 and SBS-S4; PS content about $30 \%$ ) leads to an increase in overall PS content. Thus the property measured is also partly contributed by the morphological changes. As in the previous case, the tensile properties of the SBS 
copolymers were affected only insignificantly upon addition of up to $30 \mathrm{wt} .-\%$ of the SB diblock copolymer (results not discussed here and shall be discussed in a separate paper).

\section{Dynamic mechanical properties}

Dynamic mechanical analysis (DMA) allows a good comparison of mechanical properties of the block copolymer blends in the viscoelastic region. Especially, the storage modulus or the viscoelastic loss (tangent delta) is of particular interest.

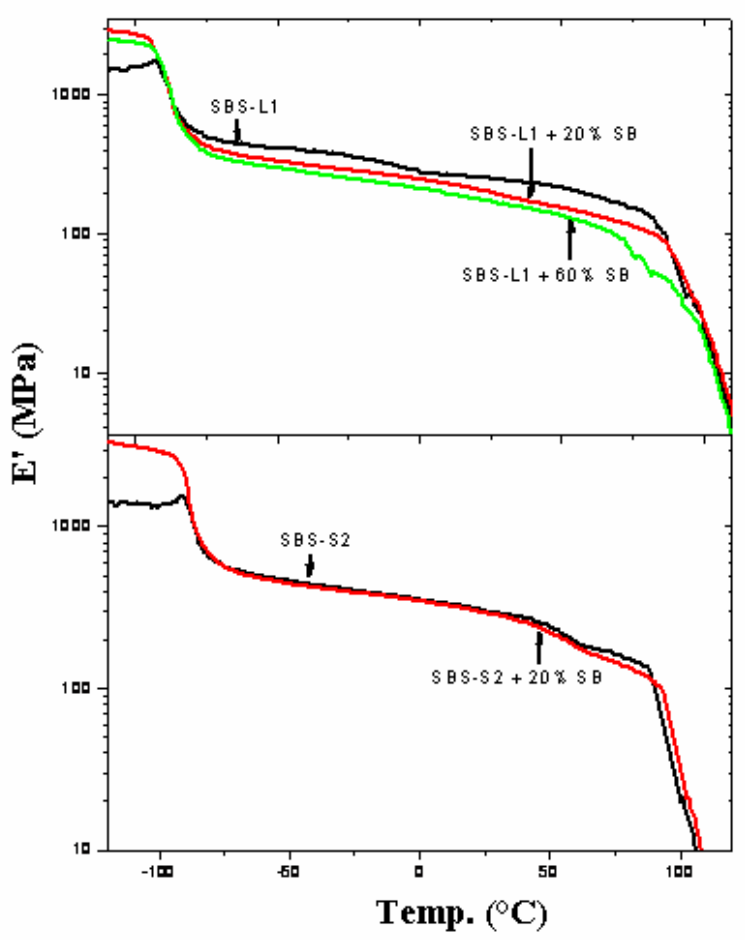

Fig. 7. Storage modulus versus temperature curves for some of binary block copolymer blends; test frequency $1 \mathrm{~Hz}$ : a) SBS-L1/SB blends and b) SBS-S2/SB blends.

Previous studies on binary triblock/diblock blends have shown that a pronounced decrease in storage modulus in the plateau region (or a corresponding increase in tangent delta) occurs on adding SB (or SI) molecules to the SBS (or SIS) triblock copolymers (Lovisi et al. 1998, McKay et al. 1995). The decrease in storage modulus is a result of diblock copolymer acting as dangling molecule, not coupled to the SBS triblock architecture.
Figure 7a compares the storage modulus of the linear block copolymer (SBS-L1) with that of its blends with 20 and 60 wt.- $\%$ of added SB diblock. In the blends, the storage modulus in the plateau region decreases with increase in SB content. The storage modulus drops by up to $100 \mathrm{MPa}$ in presence of $20 \mathrm{wt} .-\% \mathrm{SB}$ diblock. Such a decrease in storage modulus is undesirable for the application of SBS block copolymers as asphalt pavement binders or as hot melt adhesives. Astonishingly, only a small change in storage modulus was observed for the corresponding blends of SB diblock with the radial block copolymer SBS-S2 (for instance, see Figure 7b). This indicates again that the relaxation of the PB middle block is affected to a lesser extent by the diblock present in the blends with radial block copolymers.

The main conclusions of the presented works can be summarised as follows.

- Both large strain deformation tensile deformation behaviour and viscoelastic properties of the SBS block copolymers are affected considerably by the presence of large amount of uncoupled SB diblock. The storage modulus of linear SBS decreases more sharply in the plateau region than for the radial SBS at low SB content.

- At low SB content (up to 20 wt.-\% for linear SBS and still higher for radial one), the overall tensile properties are not negatively influenced. A small decrease in tensile strength is well compensated by a significant rise in elongation at break. Similar conclusion is valid for mechanical hysteresis. In overall, star block copolymers are less sensitive towards the presence of diblock.

Detailed analysis of deformation mechanisms (mainly using TEM and SEM) is underway to provide grounds for the experimental results obtained so far.

\section{Acknowledgements}

Dr. Robert E. Bening (Kraton Polymers, Houston, TX) as well as Prof. Goerg H. Michler and Prof. HansJoachim Radusch (Martin Luther University, Halle) are acknowledged for providing the polymer samples as well as laboratory facilities, respectively to conduct the studies reported in this work. 


\section{References}

Adhikari, R. 2012. Block copolymers, In: Nanomechnics of polymers, (Eds. G. H. Michler, F. J. Balta Calleja), Munich, Hanser Verlag, in Press.

Adhikari, R., S. Henning and G.H. Michler. 2010. Importance of molecular coupling for mechanical performance of block copolymer compounds. Macromoleclar Symposia 294: 55-66.

Adhikari, R. and G.H. Michler. 2004. Influence of molecular architecture on morphology and micromechanical behaviour of styrene/butadiene block copolymer systems. Progess in Polymer Science 29: 949-986.

Bates, F.S. and G.H. Fredrickson. 1990. Block copolymer thermodynamics: Theory and experiment, Annual Reviews in Physical Chemistry 41: 525-557.

Dair, B.J., C.C. Honecker, D.B. Alward, A. Avgeropoulos, N. Hadjichristidis, L.J. Fetters, M.S. Capel and E. L. Thomas. 1999. Mechanical properties and deformation behaviour of the double gyroid phase in unoriented Thermoplastic elastomers. Macromolecules 32: 8145-8152.

Davis, K.A. and K. Matyjaszewski. 2002. Statistical, gradient, block and graft copolymers by controlled/living radical polymerizations. In: Advances in polymer science 159. Berlin, Heidelberg, New York, Springer-Verlag.

Drolet, F. and G.H. Fredrickson. 1999. Combinatorial screening of complex block copolymer assembly with self-consistent field theory. Physical Reviews Letters 83: 4317-4320.
Hamley, I.W. 1998, The physics of block copolymers, Oxford, Oxford Publishers.

Holden G. 2000. Understanding thermoplastic elastomers, Munich, Hanser Verlag.

Honeker C.C. and E. L. Thomas. 2000. Perpendicular deformation of a near-single-crystal triblock copolymer with a cylindrical morphology 2: TEM. Macromolecules 2000 39: 9407-9417.

Lovisi, H.R., L.F. Nicolini, A. A. Ferreira and M.L.S. Martins. 1995. The influence of uncoupled styrene butadiene diblock copolymer on the physical properties of SBS thermoplastic elastomers, In ACS Symposium Series (Vol. 696): Applications of of ANionic Polymerization Research. American Chemical Society

Matsen, M.W. 2000. Equilibrium behavior of asymmetric ABA triblock copolymer melts. Journal of Chemical Physics 113: 5539-5544.

McKay, K.W., W.A. Gros and C.F. Diehl. 1995. The influence of styrene-butadiene diblock copolymer on styrene-butadiene-styrene triblock copolymer viscoelastic properties and product performance, Journal of Applied Polymer Science. 56: 947-958.

Milner, S.T. 1994. Chain architecture asymmetry in copolymer microphases. Macromolecules 27 :23332335.

Quirk, R.P. and M. Morton. 1998. Research on anionic triblock copolymers. In: Thermoplastic Elastomers G. Holden, N.R. Legge, R.P. ( $2^{\text {nd }}$ Edition). Munich, Hanser Publishers. 
Nepal Journal of Science and Technology 12 (2011) 149-156 